\title{
El índice de anisidina como medida del deterioro latente de un material graso.
}

\author{
Por M. A. Grompone \\ Facultad de Quimica. Casilla de Correo 1157. Montevideo, URUGUAY.
}

\section{RESUMEN}

El índice de anisidina como medida del deterioro latente de un material graso.

A pesar de que el indice de peróxido es una medida corriente de la oxidación de los lipidos, su uso está limitado a las etapas iniciales de dicha reacción. Como los peróxidos pueden sufrir descomposiciones posteriores, la historia oxidativa completa del aceite no se conoce por ellos. Se considera que el índice de anisidina, una medida de los productos de la oxidación secundaria, es muy útil para evaluar el pasado del aceite.

Se estudiaron los efectos del almacenamiento acelerado de muestras comerciales sobre los indices de anisidina y de peróxido. Los resultados obtenidos indican que el índice de anisidina es un buen método para predecir dicha estabilidad.

Se estudiaron algunas etapas del procesamiento de las grasas y aceites comestibles. Las arcillas de blanqueo producen la descomposición de los productos de oxidación y este efectc sólo puede reducir el indice de peróxido del aceite. Se desarrollaron estudios comparativos para determinar los efectos del blanqueo sobre el índice de anisidina.

El análisis de aceites deteriorados antes y después de la desodorización mostró que tiene lugar solamente una pequeña reducción en el indice de anisidina.

PALABRAS-CLAVE: Almacenamiento - Decoloración - Estabilidad - Grasa comestible - Indice de anisidina - Oxidación lipidica

\section{SUMMARY} fats.

Anisidine value as a measurement of the latent damage of

Although the peroxide value is a common measurement of lipid oxidation, its use is limited to the initial stages of oxidation. Since peroxides are vulnerable to further decomposition, the complete oxidative history of the oil may not be revealed. The anisidine test a measure of secondary oxidation products, is considered useful in that it shows the past history of the oil.

Effects of accelerated storage test on the anisidine and peroxide values of commercially samples were studied. The results obtained indicate that the anisidine value is a good method to predict storage stability of fats.

Some processing steps in producing edible fats and oils were studied. Bleaching clay performs decomposition of oxidation products and this effect alone would reduce the peroxide value. Comparative studies were undertaken to determine the effects of bleaching on the anisidine value.

Analysis of damaged oils before and after deodorization showed that little reduction of anisidine value occurred.
KEY-WORDS: Anisidine value - Bleaching - Edible fat - Lipid oxidation - Stability - Storage.

\section{INTRODUCCION}

La oxidación de compuestos olefínicos por el oxígeno atmosférico es importante en el desarrollo de la rancidez, en la producción de olores desagradables y en la polimerización de los aceites secantes. Los primeros productos aislables son hidroperóxidos insaturados. Estos son intermediarios transitorios que reaccionan dando, entre otros, compuestos con funciones carbonilo, los que a su vez pueden catalizar oxidaciones posteriores o descomponerse en compuestos indeseables. La calidad oxidada de los aceites tienen efectos significativos sobre su estabilidad durante el almacenamiento y sobre su valor nutricional (1), (2).

Si bien el índice de peróxido ha sido una medida corriente de la oxidación lipídica, su utilidad se limita a las etapas iniciales de la reacción. Como los hidroperóxidos sufren reacciones posteriores de descomposición, la historia oxidativa completa dé un aceite no se revela en su índice de peróxido: éste puede ser bajo porque el material es de buena calidad o porque ha sido reducido por algún proceso que puede enmascarar su deterioro verdadero.

Holm y colaboradores (3) introdujeron el ensayo de la bencidina como una medida de los productos de la oxidación secundaria formados durante el procesamiento de los aceites. Debido a las propiedades cancerígenas del mencionado reactivo, se sustituyó por un ensayo similar basado en el uso de la anisidina. Este método no da un valor numérico correcto del contenido en compuestos con funciones carbonilo ni una medida directa del sabor de una grasa ni de su estabilidad. Esto se debe a que no todos los compuestos carbonilo contribuyen de igual manera. Sin embargo este análisis ha resultado muy útil, a pesar de la poca frecuencia con que se emplea (47). 
El procedimiento analítico ha sido oficializado por la International Union of Pure and Applied Chemistry (8). El índice de anisidina se define, por convención, como 100 veces la absorbancia medida a $350 \mathrm{~nm}$ en una celda de un $\mathrm{cm}$ de espesor, de una solución que contiene $1.00 \mathrm{~g}$ de aceite en $100 \mathrm{ml}$ de una mezcla de solvente y reactivo, de acuerdo con el método descrito. El principio de éste radica en la reacción entre los compuestos aldehídicos y la $p$-anisidina ( $p$ metoxianilina). La intensidad del color de los productos amarillentos formados en la reacción depende no solamente de la cantidad de compuestos aldehídicos presentes sino también de su estructura: un doble enlace en la cadena carbonada, conjugado con el doble enlace del carbonilo, aumenta la absorbancia molar en 4 ó 5 veces. Esto significa que los 2-alquenales contribuyen prioritariamente al valor determinado.

Si bien hay algunos artículos que emplean el índice de anisidina como una medida del deterioro del aceite (9-11), aún no se ha sistematizado su empleo por lo que es escasa la información de referencia.

Este trabajo utiliza el índice de anisidina en comparación con el respectivo índice de peróxido, para establecer criterios de estabilidad de los materiales grasos. Estudia también las variaciones simultáneas de ambos índices en operaciones de fábrica que tienen por objetivo el mejorar la calidad de los productos, tales como el blanqueo y la desodorización.

\section{PARTE EXPERIMENTAL}

\subsection{Procedimientos empleados.}

En el laboratorio se efectuaron blanqueos con dos tipos diferentes de productos comerciales:

a) TONSIL optimun extra (Química SUMEX, México): Se trata de una tierra de blanqueo del tipo bentonita (arcilla activada).

b) Tri Syl (de W. R. Grace and Co,. USA): Se trata de una silicagel amorfa, especialmente indicada para eliminar jabones $y$ fosfolípidos.

El material graso se calentó a $100^{\circ} \mathrm{C}$ bajo vacío durante 30 minutos con la tierra de blanqueo incorporada. Transcurrido dicho tiempo y mantenido el vacío, se enfrió por debajo de $60^{\circ} \mathrm{C}$; luego se filtró sobre papel.

De acuerdo con la bibliografía (13), se efectuaron ensayos de envejecimiento acelerado con muestras destapadas puestas en estufa a $60^{\circ} \mathrm{C}$ (ensayo de Schaal).

\subsection{Métodos analiticos}

Las ténicas analíticas empleadas fueron: (14).

a) Indice peróxido ( $I_{\text {perox }}$ ): Método AOCS Cd 8-53 b) Indice de anidisina ( $\left.I_{\text {anis }}\right)$ : Método IUPAC 2.504 (8). Se utilizó un espectrofotómetro Shimadzu UV-160 con registrador.

\section{RESULTADOS Y DISCUSION}

Los ensayos realizados se pueden agrupar de la siguiente manera:

\subsection{Determinación de la estabilidad en función} del $I_{\text {perox }} y$ del $I_{\text {anis. }}$

Se estudió la estabilidad de tres aceites de soya parcialmente hidrogenados (I.I. = 95). Los tres contenían 100 ppm de BHT, 100 ppm de BHA y 100 ppm de ácido cítrico, como conservadores. Los aceites fueron elegidos de modo que inicialmente tuvieran $I_{p e}$. rox similares y muy bajos e $I_{\text {anis }}$ diferentes, con el objetivo de determinar exclusivamente la influencia de este último sobre la estabilidad. Se empleó a tales efectos el ensayo acelerado de Schaal. Se sacaron muestras cada cuatro días y se determinaron los índices correspondientes. En la Figura 1 se resumen los resultados obtenidos. Se concluye que cuanto menor es el $I_{\text {anis }}$ inicial, más dias demora el aceite colocado a $60^{\circ} \mathrm{C}$ para que su $I_{\text {perox }}$ aumente de manera importante. Es decir el período de inducción depende notoriamente del $l_{\text {anis }}$ del aceite de partida. Esto remarca el papel fundamental que tiene este indice sobre el comportamiento posterior del aceite y confirma que el $I_{\text {perox }}$ que es útil como medida del grado de deterioro o enranciamiento actual, no es un parámetro adecuado para evaluar su estabilidad o predecir su evoluciòn futura.

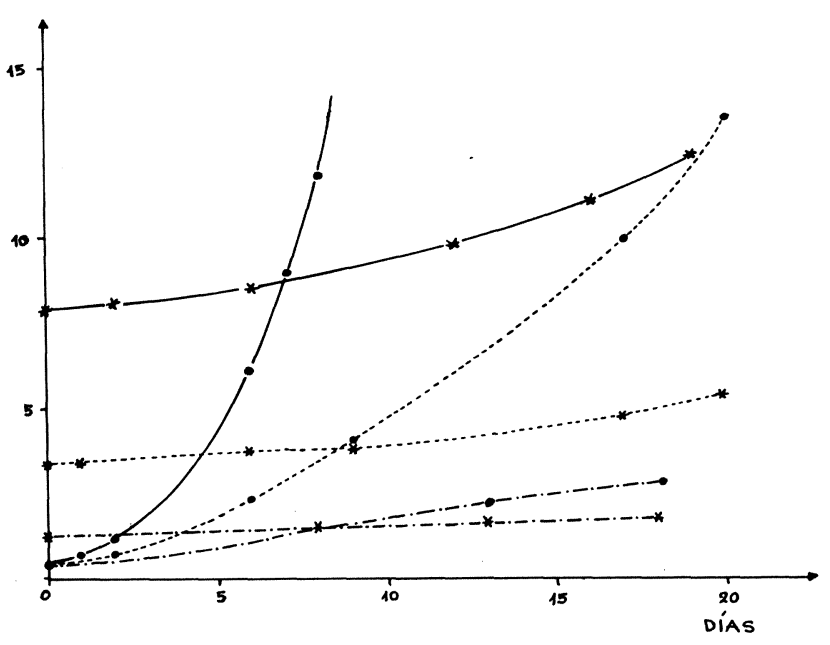

Figura 1

Aceites de soya parcialmente hidrogenados (1.I.= 95), sometidos a estufa a $60^{\circ} \mathrm{C}$.

- indice de peróxido.

- indice de anisidina.

- muestra $A$.

..... muestra $B$.

-. - muestra C. 


\subsection{Influencia de los antioxidantes.}

A una parte de una misma muestra $D$ de grasa vacuna desodorizada, se le agregó 200 ppm de BHT y 100 ppm de ácido cítrico; a la otra no se le efectuó ningún tipo de agregado. Ambas fueron colocadas simultáneamente en estufa a $60^{\circ} \mathrm{C}$ (ensayo de Schaal). En la tabla I se resumen los resultados obtenidos, de los cuales surge la influencia del antioxidante usado: a pesar del alto valor inical del $I_{\text {anis' }}$ tanto este como el $I_{\text {perox }}$ aumentan lentamente en comparación con los de la muestra que carece de antioxidantes

Tabla I

Grasa vacuna desodorizada, con y sin antioxidantes, colocada en estufa a $60^{\circ} \mathrm{C}$

\begin{tabular}{c|c|c|c|c|} 
Dias & \multicolumn{2}{|c|}{ Sin antioxidante } & \multicolumn{2}{c|}{ Con antioxidante } \\
& $I_{\text {perox }}$ & $I_{\text {anis }}$ & $I_{\text {perox }}$ & $I_{\text {anis }}$ \\
\hline 0 & 0.4 & 7.0 & 0.6 & 6.7 \\
4 & 11.0 & 10.3 & 0.8 & 6.7 \\
11 & 28.8 & 14.6 & 3.6 & 7.0 \\
22 & - & - & 6.4 & 7.9
\end{tabular}

En la tabla II se indican los valores obtenidos para tres muestras diferentes de aceite de soya, sometidas al ensayo de Schaal. La muestra $E$ corresponde a un aceite refinado y desodorizado que no contiene antioxidantes; la muestra $F$ a un aceite crudo (semirefinado) $\sin$ antioxidantes y la muestra $G$, a un aceite refinado y desodorizado con agregado de antioxidantes.

\section{Tabla II}

Muestras de aceite de soya sometidas a estufa a $60^{\circ} \mathrm{C}$. Muestra E: aceite refinado y desodorizado sin antioxidantes. Muestra F: aceite crudo sin antioxidantes. Muestra G: aceite refinado y desodorizado con antioxidantes.

\begin{tabular}{c|c|c|c|c|c|c|}
\multirow{2}{*}{ Dias } & \multicolumn{2}{|c|}{ Muestra E } & \multicolumn{2}{c|}{ Muestra F } & \multicolumn{2}{|c|}{ Muestra G } \\
& $I_{\text {perox }}$ & $I_{\text {an1s }}$ & $I_{\text {perox }}$ & $I_{\text {an1s }}$ & $I_{\text {perox }}$ & $I_{\text {anis }}$ \\
\hline 0 & 1.4 & 0.7 & 2.3 & 1.9 & 1.5 & 8.6 \\
6 & 14.4 & 2.3 & 19.3 & 3.4 & 5.3 & 8.8 \\
10 & - & - & 36.8 & 5.7 & 7.9 & 8.8 \\
14 & 43.0 & 7.0 & 60.6 & 9.1 & 11.8 & 8.9 \\
22 & 94.8 & 15.0 & - & - & 15.8 & 9.4 \\
29 & 124.0 & 19.3 & - & - & 42.3 & 9.7
\end{tabular}

A pesar de que las muestras $E$ y $F$ (que no contienen antioxidantes) presentan valores iniciales bajos de $I_{\text {perox }} \theta I_{\text {anis' }}$, no son estables en la estufa a $60^{\circ} \mathrm{C}$ ya que ambos índices aumentan considerablemente en pocos días. Por el contrario, la muestra G (que contiene antioxidantes), presenta un período de inducción importante, a pesar del alto valor inicial de su $I_{\text {anis }}$. Este varía muy poco con el tiempo de estadía en estufa, comportamiento diferente también al de las muestras $E$ y $F$.

La elección de muestras a efectos comparativos debe ser cuidadosamente hecha porque son muchos y variados los factores que influyen sobre la estabilidad de las grasas. Las muestras D (grasa vacuna) y las $E, F$ y $G$ (aceite vegetal) no deben ser comparadas debido a su diferente composición química, ya que cuanto mayor es el grado de insaturación del material graso, más susceptible es éste al deterioro oxidativo. Los antioxidantes primarios son sustancias que funcionan inhibiendo o interrumpiendo el mecanismo de radicales libres de la autoxidación de los glicéridos. Sin embargo, ningún antioxidante es la panacea para el deterioro oxidativo de todos los productos alimenticios. La selección del antioxidante apropiado está determinada por el tipo de material graso y del uso que de éste se haga. Así tanto el BHT como el BHA son especialmente indicados para prevenir la oxidación en grasas animales pero son menos efectivos en aceites vegetales (1) (2) (15). A esto puede deberse las diferencias de estabilidad en condiciones similares mostradas en las Tablas I y II $y$ en la Figura 1.

\subsection{Influencia del blanqueo.}

Se hicieron blanqueos en el laboratorio con $2 \%$ de TriSyl y con $2 \%$ de Tonsil, separadamente, sobre un aceite de soya crudo (semi-refinado). Cada muestra se dividió en dos partes: sobre una se efectuó el ensayo de Schaal, la otra permaneció tapada a temperatura ambiente y expuesta a la luz natural. Los resultados obtenidos se informan en la Tabla III.

Tanto las determinaciones sobre las muestras puestas en estufa como las dejadas a temperatura ambiente indican que el blanqueo de un aceite crudo no mejora sensiblemente su estabilidad. Se nota inclusive un efecto negativo del efectuado con Tonsil ya que si bien el $l_{\text {perox }}$ es menor que el inicial su $l_{\text {anis }}$ se ve incrementado. En conclusión, el blanqueo al inicio de las operaciones de refinación de un aceite no mejora su estabilidad futura si éste ya ha comenzado su proceso de deterioro.

En la Tabla IV se resumen los ensayos de blanqueo efectuados con muestras diferentes que presen$\tan$ relaciones variables entre los $I_{\text {perox }} \otimes I_{\text {anis }}$ 
Tabla III

Aceite de soya crudo blanqueado con TriSyl y con Tonsil y sometido a un proceso de envejecimiento natural y en estufa a $60^{\circ} \mathrm{C}$.

\begin{tabular}{|c|c|c|c|c|c|c|}
\hline \multirow[t]{2}{*}{ Dias } & \multicolumn{2}{|c|}{ Sin blanquear } & \multicolumn{2}{|c|}{$2 \%$ Tonsil } & \multicolumn{2}{|c|}{$2 \%$ Trisy 1} \\
\hline & $I_{\text {perox }}$ & $I_{\text {anis }}$ & $I_{\text {perox }}$ & $I_{\text {anis }}$ & $I_{\text {perox }}$ & $I_{\text {anis }}$ \\
\hline 0 & 2.3 & 1.9 & 0.6 & 4.6 & 3.2 & 1.9 \\
\hline ESTUFA: & & & & & & \\
\hline 6 & 19.3 & 3.4 & 21.8 & 6.5 & 25.2 & 4.2 \\
\hline 10 & 36.8 & 5.7 & 36.7 & 9.2 & 36.8 & 6.3 \\
\hline 14 & 60.6 & 9.1 & 58.5 & 11.9 & 59.5 & 9.5 \\
\hline AMBIENTE & & & & & & \\
\hline 12 & 6.8 & 2.2 & 3.6 & 4.6 & 7.6 & 2.1 \\
\hline 19 & 9.1 & 2.2 & 10.4 & 4.6 & 10.4 & 2.1 \\
\hline 31 & 14.0 & 2.4 & 12.8 & 4.8 & 17.0 & 2.2 \\
\hline 47 & 20.4 & 2.5 & 23.2 & 4.8 & 28.5 & 2.2 \\
\hline
\end{tabular}

Tabla IV

Ensayos de blanqueo con diferentes muestras de materiales grasos.

\begin{tabular}{|c|c|c|c|c|}
\hline Muestra & $\%$ Trisyl & $\%$ Tonsil & $I_{\text {perox }}$ & $I_{\text {anis }}$ \\
\hline \multirow[t]{3}{*}{ a } & - & - & 0.3 & 1.3 \\
\hline & 2.0 & - & 0.6 & 1.2 \\
\hline & - & 2.0 & 0.4 & 1.3 \\
\hline \multirow[t]{3}{*}{$\mathrm{b}$} & - & - & 2.3 & 1.9 \\
\hline & 2.0 & - & 3.2 & 1.9 \\
\hline & - & 2.0 & 0.6 & 4.6 \\
\hline \multirow[t]{7}{*}{ c } & - & - & 0.3 & 8.1 \\
\hline & 1.0 & - & 0.4 & 7.7 \\
\hline & 2.0 & - & 0.6 & 7.1 \\
\hline & 4.5 & - & 0.6 & 6.5 \\
\hline & - & 1.0 & 0.4 & 6.8 \\
\hline & - & 2.0 & 0.6 & 6.0 \\
\hline & - & 4.5 & 0.4 & 3.4 \\
\hline \multirow[t]{5}{*}{$d$} & - & - & 1.6 & 22.9 \\
\hline & 2.0 & - & 2.9 & 20.0 \\
\hline & 4.5 & - & 3.0 & 18.1 \\
\hline & - & 2.0 & 0.2 & 16.9 \\
\hline & - & 4.5 & 0.3 & 9.9 \\
\hline \multirow[t]{6}{*}{ e } & - & - & 17.4 & 6.3 \\
\hline & 2.0 & - & 15.2 & 6.8 \\
\hline & 4.5 & - & 6.8 & 10.9 \\
\hline & - & 2.0 & 1.2 & 22.3 \\
\hline & - & 4.5 & 0.4 & 13.7 \\
\hline & 1.5 & 3.0 & 0.6 & 15.9 \\
\hline
\end{tabular}

La muestra a presenta $I_{\text {perox }} \otimes I_{\text {anis }}$ muy bajos: tanto su blanqueo con Tonsil como con TriSyl no tienen efecto apreciable sobre ambos parámetros.

La muestra b presenta un $I_{\text {perox }}$ un poco más alto que la anterior y un $I_{\text {anis }}$ similar: el blanqueo con TriSyl no tiene efectos importantes pero el efectuado con Tonsil aumenta considerablemente el $l_{\text {anis }}$ bajando simultáneamente su $I_{\text {perox. }}$

La muestra c presenta un $\mathrm{I}_{\text {perox }}$ muy bajo y un $\mathrm{I}_{\text {anis }}$ alto. Los blanqueos con diferentes cantidades de TriSyl modifican muy poco esos valores. El blanqueo con $4.5 \%$ de Tonsil disminuye a menos de la mitad el $I_{\text {anis }}$ sin afectar su $I_{\text {perox. }}$ Esto mejora la estabilidad del aceite de acuerdo con lo observado en la Figura 1.

La muestra d presenta un $I_{\text {perox }}$ un poco más alto que el de la muestra $c$ y un $l_{\text {anis }}$ muy alto. El comportamiento es similar al observado en la muestra $c$ ya que, tanto con TriSyl como con Tonsil, el $I_{\text {anis }}$ disminuye al aumentar la cantidad de blanqueador usado. Respecto al I perox' el TriSyl lo aumenta pero el Tonsil lo disminuye.

La muestra e presenta un $\mathrm{I}_{\text {perox }}$ muy alto y un $\mathrm{I}_{\text {anis }}$ alto también. Los blanqueos con TriSyl y con Tonsil disminuyen el $\mathrm{I}_{\text {perox }}$ (siendo el efecto mucho más importante para el segundo) y aumentan el $\mathrm{I}_{\text {anis }}$ respecto a los del material de partida. Para los blanqueos efectuados con Tonsil se observa que el $I_{\text {anis }}$ correspondiente al $2 \%$ es mucho mayor que el correspondiente al $4.5 \%$, es decir que, comparativamente, éste disminuye al aumentar la proporción de tierra de blanqueo usada (efecto similar al observado en la muestra d). En cambio, al aumentar la cantidad de TriSyl, el $I_{\text {perox }}$ disminuye poco y el $I_{\text {anis }}$ aumenta. El efecto combinado de ambos blanqueadores no mejora los resultados.

Este comportamiento respecto al blanqueo, que a primera vista parece no seguir una regla definida, puede ser explicado en función de las reacciones que tienen lugar en el sistema.

De acuerdo con la literatura (12) (16) (17) la remoción del color durante el blanqueo se asocia no sólo con la adsorción física sino también con un proceso que involucra la quimisorción y la subsecuente reacción química en la superficie de la arcilla activada. La función primaria del blanqueo es descomponer los productos de la oxidación tales como peróxidos y secundariamente, el proceso elimina trazas de jabón y fosfátidos remanentes de la refinación y los pigmentos que dan color.

Para explicar estos fenómenos se puede recurrir al siguiente esquema de reacciones, en el cual los hidroperóxidos conjugados se pueden descomponer dando aldehídos, cetonas y ácidos de bajo y medio peso molecular, saturados $e$ insaturados $y$ otros productos oxigenados bifuncionales (disminuyendo así el perox $_{\text {p: }}$ 


$$
\begin{gathered}
-\mathrm{CH}=\mathrm{CH}-\mathrm{CH}=\mathrm{CH}-\mathrm{CH}-\mathrm{CH}_{2}-\underset{\text { arcilla }}{\mathrm{O}_{\mathrm{OH}}} \\
\text { activada }
\end{gathered}\left[\begin{array}{c}
0 \\
\mathrm{II} \\
-\mathrm{CH}=\mathrm{CH}-\mathrm{CH}=\mathrm{CH}-\mathrm{C}-\mathrm{CH}_{2}-+\mathrm{H}_{2} \mathrm{O} \\
-\mathrm{CH}=\mathrm{CH}-\mathrm{CH}=\mathrm{CH}-\mathrm{CH}=\mathrm{CH}-+\mathrm{H}_{2} \mathrm{O}
\end{array}\right.
$$

Los productos de la oxidación secundaria también pueden descomponerse a nivel de la arcilla activada, disminuyendo así el $l_{\text {anis }}$. El conjunto de estas reacciones puede esquematizarse en la siguiente secuencia:

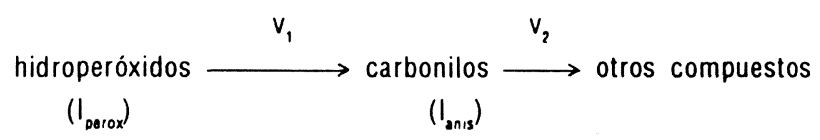

Cuando los hidroperóxidos responsables de la oxidación primaria se descomponen formando carbonilos, se produce una disminución del $\mathrm{I}_{\text {perox }}$ y un aumento del $I_{\text {anis }}$ (muestra e con $2 \%$ de Tonsil). Si la cantidad de tierra de blanqueo es suficientemente grande también se logra eliminar productos de la ruptura oxidativa, disminuyendo comparativamente el $\mathrm{I}_{\text {anis }}$ (muestra e con $4.5 \%$ de Tonsil). Si la relación de velocidades de reacción es $v_{1}>v_{2}$ se forman los carbonilos más rápido de lo que se descomponen y el $\mathrm{I}$ an aumenta; si $v_{1}$ " $v_{2}$ debido, por ejemplo a que el contenido en peróxidos es chico, el $l_{\text {anis }}$ decrece (muestra c con Tonsil).

De esta manera, el efecto neto que produce el blanqueo con arcillas activadas depende del contenido en hidroperóxidos (medido en función del $\left.\right|_{\text {perox }}$ ) y de los productos secundarios de la ruptura oxidativa (medidos como $I_{\text {anis }}$ ). Los carbonilos sólo pueden ser eliminados parcialmente en el blanqueo con arcillas activadas, ya que no son removibles de manera apreciable por procesos de desodorización porque en general no son volátiles. En cambio, los peróxidos son térmicamente lábiles y se destruyen en las condiciones de la desodorización (18). Por estas razones, el $I_{\text {perox }}$ puede disminuir en la desodorización sin afectar apreciablemente el $I_{\text {anis }}$.

\subsection{Influencia de la desodorización.}

Se estudiaron muestras de grasa vacuna y de aceite de soya en diferentes etapas de su procesamiento a nivel de planta industrial. En la Tabla $V$ se indican los resultados obtenidos.

La hidrogenación catalítica baja considerablemente los índices considerados pero la desodoriza- ción sólo afecta apreciablemente al $\mathrm{I}_{\text {perox }}$. Esta operación no resulta eficaz para eliminar la oxidación secundaria, una vez que ésta tiene lugar.

\section{Tabla V}

Grasa vacuna y aceite de soya procesados a nivel de fábrica.

\begin{tabular}{l|c|c|c|c|} 
& \multicolumn{2}{|c|}{ Grasa vacuna } & \multicolumn{2}{c|}{ Aceite de soya } \\
& $I_{\text {perox }}$ & $I_{\text {anis }}$ & $I_{\text {perox }}$ & $I_{\text {anis }}$ \\
\hline $\begin{array}{l}\text { antes de hidro- } \\
\text { genar }\end{array}$ & - & - & 13.0 & 9.0 \\
$\begin{array}{l}\text { parcialmente } \\
\text { h1drogenada } \\
\text { (I.I.=76) }\end{array}$ & - & - & & \\
$\begin{array}{l}\text { blanqueada } \\
\text { desodorizada }\end{array}$ & 5.4 & 6.8 & 1.0 & 4.2 \\
& 0.4 & 5.5 & 0.8 & 6.3 \\
& & & 0.6 & 4.0
\end{tabular}

\section{CONCLUSIONES}

Un aceite puede presentar un $I_{\text {perox }}$ bajo debido a su buena calidad de origen 0 al tratamiento a que fue sometido. Este valor, por lo tanto, no es garantía de buena estabilidad de almacenamiento. El $\mathrm{l}_{\text {anis }}$ es un parámetro adecuado para evaluar su historia y prever su comportamiento futuro. Cuanto mayor sea su $I_{\text {anis }}$ menor será su tiempo de inducción, o sea, menor el período de tiempo en el que esencialmente no hay oxidación y por lo tanto, la formación de peróxidos es nula o muy pequeña. Cuanto mayor sea el $l_{\text {anis }}$ más rapidamente comenzará la autoxidación y se enranciará el material graso. El uso de antioxidantes adecuados mejora la estabilidad.

La desodorización disminuye considerablemente el $I_{\text {perox }}$ pero afecta poco al $I_{\text {anis }}$ con lo cual no se mejora la estabilidad de un material inicialmente deteriorado.

El blanqueo con arcillas activadas suele disminuir el I perox también. (EI blanqueo con TriSyl suele aumentarlo, pero se trata de una silicagel amorfa que se usa especialmente para eliminar jabones y fosfoglicéridos). Su efecto sobre el $I_{\text {anis }}$ depende del $I_{\text {perox }}$ del material en cuestión: con una cantidad adecuada de tierra de blanqueo se puede disminuir el $I_{\text {anis }}$ pero si el material graso está muy alterado $\left(I_{\text {perox }}\right.$ e $i_{\text {anis }}$ muy altos) esto ya no es posible. Un aceite dañado de origen difícilmente puede ser utilizado para obtener un producto final de buena calidad, estable durante su almacenamiento. 
BIBLIOGRAFIA

1. Gunstone, F. D. y Norris, F. A.- "Oxidation", capitulo 7 de "Lipids in foods".- Pergamon Press, Oxford, 1983

2. Sherwin, E. R.- "Oxidation and antioxidants in fat and oil processing".- J. Am. Oil Chemists'Soc. 55 (1978) 809-814.

3. Holm, U., Ekbom K. y Wobe, G.- "Determination of the extent of oxidation of fats". - J. Am. Oil Chemists 'Soc. 34 (1957) 606-609.

4. List, G. R., Evans, C. D., Kwolek, W. F., Warner, K., Boundy B. K. y Cowan, J. C.- "Oxidation and quality of soybean oil: a preliminary study of the anisidine test".- J. Am. Oil Chemists Soc. 51 (1974) 17-21.

5. Tsoukalas, B. y Grosch, W.- "Analysis of fat deterioration: Comparison of some photometric tests".- J. Am. Oil Chemists Soc 54 (1977) 490-493.

6. Gray, J. I.- "Measurement of lipid oxidation: a review".- J. Am. Oil Chemists'Soc. 55 (1978) 539-546.

7. Hung, S. S. O. y Slinger, S. J.- "Studies of chemical methods for assessing oxidative quality and storage stability of feeding oils".- J. Am. Oil Chemists'Soc. 58 (1981) 785-788.

8. Paquot, C. y Hauffenne, A. (Editores)- "Standard methods for the analysis of oils, fats and derivates".- 7th. Revised and enlarged edition. International Union of Pure and Applied Chemistry (IUPAC). Commission on Oils, Fats and Derivatives.Blackwell Sci. Pub., Oxford, 1987.

9. Cornelius, J. A.- "International standards for palm oil".- J. Am. Oil Chemists'Soc. 54 (1977) 943 A - 948 A.

10. Strecker, L. R., Hasman, J. M. y Maza, A.- "Quality characteristics and properties of the principal world oils when processed by physical refining".- Proceeding: World Conference on emerging technologies in the fats and oils industry.- A. R. Baldwing (editor).- American Oil Chemists Society, Champaign, Illinois. 1986.

11. Ungermann, C. B.- "Effects of phospholipid content on refined soybean oil quality".- Lecture presented at the AOCS Annual Meeting, Hawaii, 1986.

12. Nkpa, N. N.; Arowolo, T. A. y Akpan, H. J.- "Quality of Nigerian palm oil after bleaching with local treated clays".- J. Am. Oil Chemists' Soc. 66 (1989) 218-222.

13. Sherwin, E.R.- "Methods for stability and antioxidant measurement".- J. Am. Oil Chemists'Soc. 45 (1968) 632 A - 649 A

14. Walker, R. C. (Editor).-- "Official Methods and Recommended Practices of the American Oil Chemists' Society".- AOCS Champaign, 1986.

15. Dziezak, J. D.- "Preservatives: Antioxidants. The ultimate answer to oxidation".- Food Technol. 40 (1986) 94 - 102.

16. Wiedermann, L. H.- “Degumming, refining and bleaching soybean oil".- J. Am. Oil Chemists' Soc. 58 (1981) 159 - 166.

17. Hastert, R. C.- "Effective adsorptive treatment of edible oils".(Technical presentation).- AOCS short course.- Phoenix, Arizona, 1988.

18. Swoboda, P.A.T.- "Chemistry of refining".- J. Am. Oil Chemists' Soc. 62 (1985) 287.

(Recibido: Abril 1990) 